\title{
SIGNED TOTAL ROMAN EDGE DOMINATION IN GRAPHS
}

\author{
Leila Asgharsharghi \\ AND \\ Seyed Mahmoud Sheikholeslami \\ Department of Mathematics \\ Azarbaijan Shahid Madani University \\ Tabriz, I.R. Iran \\ e-mail: \{l.sharghi;s.m.sheikholeslami\}@azaruniv.edu
}

\begin{abstract}
Let $G=(V, E)$ be a simple graph with vertex set $V$ and edge set $E$. A signed total Roman edge dominating function of $G$ is a function $f: E \rightarrow$ $\{-1,1,2\}$ satisfying the conditions that (i) $\sum_{e^{\prime} \in N(e)} f\left(e^{\prime}\right) \geq 1$ for each $e \in$ $E$, where $N(e)$ is the open neighborhood of $e$, and (ii) every edge $e$ for which $f(e)=-1$ is adjacent to at least one edge $e^{\prime}$ for which $f\left(e^{\prime}\right)=2$. The weight of a signed total Roman edge dominating function $f$ is $\omega(f)=$ $\sum_{e \in E} f(e)$. The signed total Roman edge domination number $\gamma_{s t R}^{\prime}(G)$ of $G$ is the minimum weight of a signed total Roman edge dominating function of $G$. In this paper, we first prove that for every tree $T$ of order $n \geq 4$, $\gamma_{s t R}^{\prime}(T) \geq \frac{17-2 n}{5}$ and we characterize all extreme trees, and then we present some sharp bounds for the signed total Roman edge domination number. We also determine this parameter for some classes of graphs.

Keywords: signed total Roman dominating function, signed total Roman domination number, signed total Roman edge dominating function, signed total Roman edge domination number.
\end{abstract}

2010 Mathematics Subject Classification: 05C69.

\section{REFERENCES}

[1] H.A. Ahangar, J. Amjadi, S.M. Sheikholeslami, L. Volkmann and Y. Zhao, Signed Roman edge domination numbers in graphs, J. Comb. Optim. 31 (2016) 333-346. doi:10.1007/s10878-014-9747-8 
[2] T.W. Haynes, S.T. Hedetniemi and P.J. Slater, Fundamentals of Domination in Graphs (Marcel Dekker, New York, 1998).

[3] T.W. Haynes, S.T. Hedetniemi and P.J. Slater, Domination in Graphs: Advanced Topics (Marcel Dekker, New York, 1998).

[4] H. Karami, A. Khodkar and S.M. Sheikholeslami, Lower bounds on signed edge total domination numbers in graphs, Czechoslovak Math. J. 58 (2008) 595-603. doi:10.1007/s10587-008-0038-3

[5] V.R. Kulli and D.K. Patwari, On the edge domination number of a graph, in: Proc. of the Symp. on Graph Theory and Combinatorics (Cochin, 1991), Centre Math. Sci. (Trivandrum, 1991) 75-81.

[6] W. Song, L. Miao, H. Wang and Y. Zhao, Maximal matching and edge domination in complete multipartite graphs, Int. J. Comput. Math. 91 (2014) 857-862. doi:10.1080/00207160.2013.818668

[7] L. Volkmann, Signed total Roman domination in graphs, J. Comb. Optim. 32 (2016) 855-871. doi:10.1007/s10878-015-9906-6

[8] D.B. West, Introduction to Graph Theory (Second Edition) (Prentice Hall, USA, 2001).

[9] B. Xu, On signed edge total domination numbers of graphs, J. East China Jiaotong Univ. 23 (2006) 129-131.

[10] J.F. Zhao and B.G. Xu, On signed edge total domination numbers of graphs, J. Math. Research \& Exposition 31 (2011) 209-214.

Received 25 April 2016

Revised 27 August 2016

Accepted 27 August 2016 